\title{
Gamificación en la evaluación dinámica de contenidos de la materia básica del Grado en Criminología
}

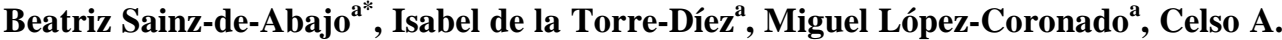 \\ Couto $^{\mathrm{a}}$ y Carlos de Castro Lozano ${ }^{\mathrm{b}}$
}

${ }^{a}$ Departamento de Teoría de la Señal, Comunicaciones e Ingeniería Telemática. Universidad de Valladolid. Paseo de Belén, 15, 47011 - Valladolid. E-mail: beasai@tel.uva.es ${ }^{*}$, isator@tel.uva.es, miglop@tel.uva.es, celso.couto@uva.es

${ }^{\mathrm{b}}$ Departamento de Informática y Análisis Numérico. Universidad de Córdoba. Carretera MadridCádiz Km.396-A, 14071-Córdoba. E-mail: ma1caloc@uco.es

\begin{abstract}
This study aims to motivate the students, transforming the learning process through gamification as a pedagogical tool, seeking to arouse students' interest in the subject, and encouraging them through a system of rewards that add up to the grade end of the course. In order to evaluate the level of previous knowledge that the student has, on the subject, we can apply this technique at the beginning of the lesson, adjusting the contents anticipated in the development of the session at this level, focusing on aspects where there are more problems of understanding. Applying the gamification in the final minutes of the lesson will allow us to evaluate the comprehension of the contents of the subject and to improve the level of attention of the student, who knows that what was presented in the session will be immediately tested at the end of the presentation.
\end{abstract}

Keywords: gamification, KAHOOT!, competition, training, self-examination, motivation, smartphones, reward.

\section{Resumen}

El objetivo de este trabajo es motivar a los alumnos, convirtiendo en lúdico el proceso de aprendizaje a través de la gamificación como herramienta pedagógica, buscando interesar a los alumnos por la materia, e incentivándoles a través de un sistema de recompensas que se sumen en la nota final del curso. Para evaluar el nivel de conocimientos previo que el alumno tiene sobre la materia, podemos aplicar esta técnica al inicio de la clase, ajustando los contenidos previstos en el desarrollo de la sesión a ese nivel, enfocándonos en los aspectos donde muestran más problemas de comprensión. Aplicar la gamificación en los minutos finales de la clase, nos permite evaluar el alcance de comprensión de los contenidos del tema y mejorar el nivel de atención del alumno, que sabe que lo expuesto en la sesión será inmediatamente testeado al finalizar la exposición.

Palabras clave: gamificación, KAHOOT!, competición, entrenamiento, autoevaluación, motivación, smartphones, recompensa. 


\section{Introducción}

La dificultad con la que los profesores nos encontramos en el aula es que la transmisión del conocimiento llegue al alumno de forma efectiva y que la interioricen a través de aprendizajes más activos. Desde el Espacio Europeo de Educación Superior (EEES) se nos insta en el uso de técnicas pedagógicas para lograr ese fin (Tejedor y García-Valcárcel, 2007). Y enfocados en ese esfuerzo las universidades nos animan a adaptar la metodología para atender a la diversidad del alumnado, entendiendo sus circunstancias y ajustando el programa para favorecer el éxito en los resultados.

En una sociedad cada vez más tecnológica, donde nuestros estudiantes manejan con gran soltura los dispositivos, parece lógico pensar en el uso de las tecnologías como método de instrucción y entrenamiento para maximizar el aprendizaje del discente. Hemos pasado de pedir a nuestros alumnos que apaguen sus dispositivos durante la clase, para evitar distracciones, a fomentar su uso. En este sentido, la importancia de este diseño pedagógico en la educación actual ha derivado en el desarrollo de toda una rama del conocimiento, englobada en el llamado m-learning, fundamentado básicamente en el uso de dispositivos móviles (smartphones, tabletas u ordenadores portátiles) en la docencia (San Miguel et al., 2017; Rodríguez-Fernández, 2017). Por ello empresas e instituciones desarrollan aplicaciones para apoyar la tecnología Bring Your Own Device (BYOD), donde los estudiantes traen un dispositivo móvil de propiedad personal para usar en cualquier lugar y momento con el propósito de aprender (Song, 2014).

La gamificación es una herramienta que aplica técnicas de juego en entornos que no son juegos o, como citan más ampliamente en internet numerosas fuentes, entre ellas el blog Proyecta (2018), “es el empleo de dinámicas y mecánicas de juego en entornos y aplicaciones no lúdicas con el fin de potenciar la motivación, la concentración, el esfuerzo, la fidelización y otros valores positivos comunes a todos los juegos”.

En pro de incentivar al alumno han aparecido numerosas aplicaciones gestoras gratuitas de participación como tendencia educativa motivadora, para la realización de test en línea que mejoran notablemente la atención de los estudiantes, siendo las más conocidas KAHOOT! (https://kahoot.com/), SOCRATIVE (https://www.socrative.com/), QUIZIZZ (https://quizizz.com/) o GOOGLEFORMS (https://www.google.com/forms/about/). En estos últimos años son muchos los trabajos que avalan de forma positiva la influencia motivadora de los juegos en el aula (Barnes, 2017; Chaiyo \& Nokham, 2017; San Miguel et al., 2017; Garcia-Garcia et al., 2017; Peña et al., 2017; Roger et al., 2017; Llerena y Rodriguez, 2017; Wang y Lieberoth, 2016). Si además a los alumnos se les premia con la posibilidad de mejorar la calificación final, si obtienen la mejor puntuación en la competición en relación al resto de compañeros, esta mecánica de juego puede ser motivadora y convertir en entretenido el proceso de aprendizaje (Iwamoto et al., 2017).

A la estrategia que se planteó en cursos pasados: visitas programadas y prácticas de laboratorio, que fueron muy valoradas por la mayoría de los alumnos, y que les permite asimilar mejor los contenidos teóricos y conceptos vistos en el aula, se suma en este curso

(cc) BY-NC-ND 2018, Universitat Politècnica de València

Congreso IN-RED (2018) 
una "nueva" tendencia educativa: la gamificación. Es necesario habilitar mecanismos para que, a pesar del absentismo que a priori se espera en el aula los días planificados como clase magistral, se alcancen los objetivos previstos adaptando la metodología.

La idea es que los alumnos que hagan el esfuerzo de acudir al aula, tengan como incentivo el reconocimiento de su asistencia, su puntualidad, su atención en clase y su colaboración en las tareas propuestas.

\section{Objetivos}

El objetivo principal de nuestra experiencia es conocer el progreso de los estudiantes en la materia, aumentar su compromiso y despertar su curiosidad.

Podemos evaluar su nivel de conocimientos previo, antes del inicio del tema, mediante un cuestionario rápido. Esto nos permitirá ajustar los contenidos de la sesión para profundizar en los puntos donde los alumnos parecen tener más dificultades.

Finalizado el tema se aplica nuevamente la gamificación, para testar el alcance de la comprensión. El discente sabe que los fallos no restan en la evaluación, por lo que se anima a participar. Igualmente sabe que puede obtener una recompensa en forma de puntos que sumen en la nota final del curso, con lo que este acicate mejora el nivel de atención, dado que lo expuesto en la sesión será evaluado al finalizar el tema. La ludificación se convierte de esta forma en una técnica entretenida que nos permite mejorar el rendimiento académico del alumno.

\section{Desarrollo de la innovación}

\subsection{Contexto}

El grupo que participó en esta metodología está integrado por un total de 17 estudiantes de la asignatura de carácter básico "Recursos Documentales e Informáticos", del Curso de Adaptación del Grado en Criminología. Se ha desarrollado en el primer cuatrimestre del curso académico 2017-18 en la Universidad de Valladolid.

Los alumnos pertenecen a las fuerzas y cuerpos de seguridad del estado (policía nacional, guardia civil y policía municipal). La media de edad ronda los cuarenta, algunos incluso los cincuenta años, y su interés es la obtención del Grado para poder ascender laboralmente (ver Fig. 1). Son inmigrantes digitales y el uso de nuevas herramientas tecnológicas y plataformas les supone un esfuerzo considerablemente mayor que a los alumnos de 18 años. Sin embargo, no tuvieron ninguna dificultad con KAHOOT!. 


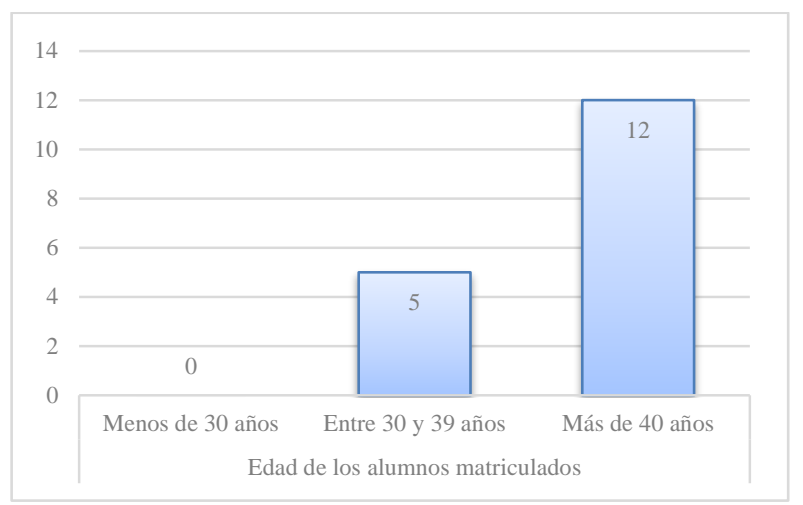

Fig.1 Edad de los alumnos que participaron en la experiencia

Se ha observado en los pasados cursos, que la gran mayoría únicamente asiste los días donde se programa una actividad que suma en la nota final de la evaluación o aquellas de asistencia obligada. En caso contrario el absentismo es generalizado. $\mathrm{Y}$ ante tanta desmotivación, ¿cómo les incentivamos?, ¿cómo podemos ayudar a gente madura que no estudia desde hace años? La solución está en ofrecer un aliciente: ¡JUGUEMOS! Y es que los seres humanos se sienten irremediablemente atraídos por los juegos.

\subsection{Procedimiento}

La aplicación de la metodología contó con una serie de fases (Fig. 2):

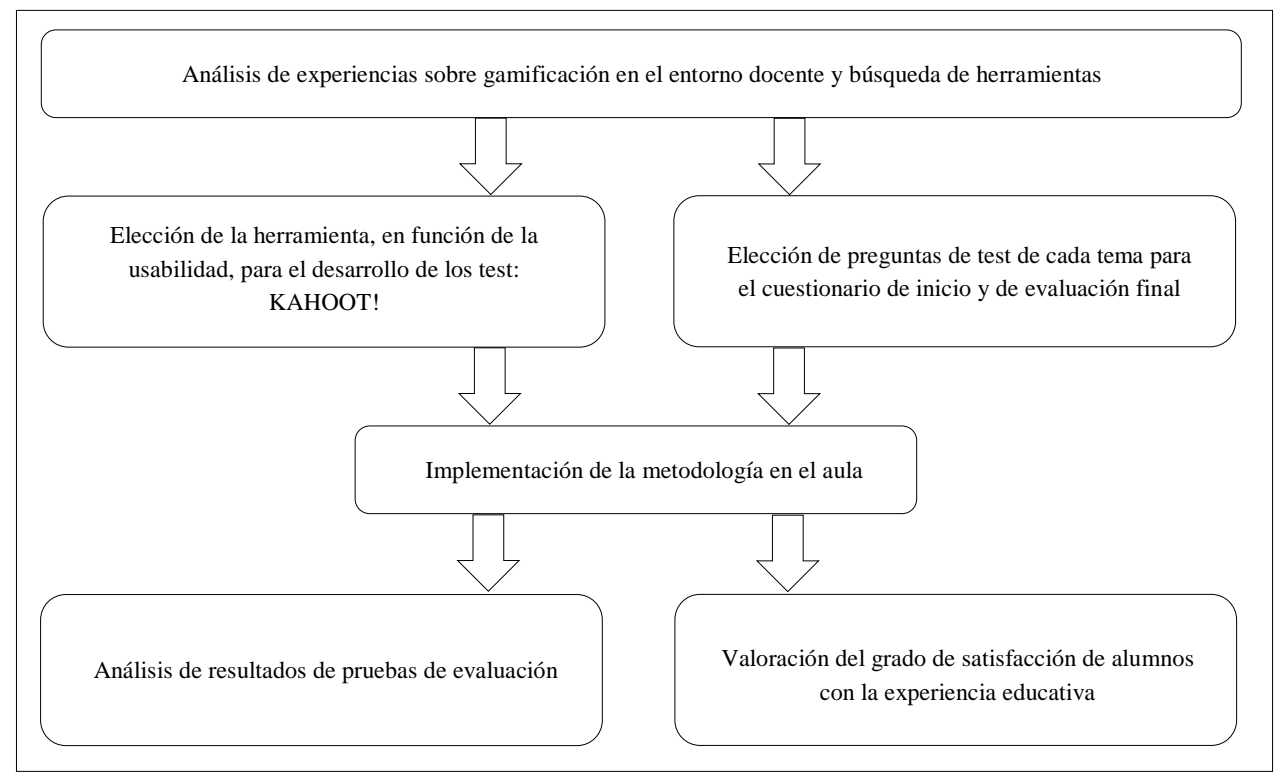

Fig. 2 Diagrama de flujo de la metodología implementada.

(cc) EY-NC-ND 2018, Universitat Politècnica de València

Congreso IN-RED (2018) 
Antes del inicio del curso se optó por gamificar con KAHOOT! para la elaboración de los test en línea, por su estética y sencillez de uso, ya que permite participar sin necesidad de conocer previamente el manejo al ser muy intuitiva. KAHOOT! es una aplicación pedagógica en continuo desarrollo que va incorporando más funcionalidades. Junto con SOCRATIVE se han convertido en una de las herramientas de gestión más populares (Fuertes et al., 2016).

Se plantearon diferentes test evaluativos para medir los conocimientos de los alumnos en relación con la materia vista. Para ello se seleccionaron un conjunto de preguntas de cada tema con diferente grado de dificultad. Los de inicio de tema contaban con 5 o 10 cuestiones, mientras que los de evaluación final incluían entre 20 y 25 cuestiones. A través de las respuestas de los alumnos y el grado de acierto se pudo valorar si los contenidos eran entendibles. Algunas de las preguntas planteadas en el test de inicio fueron incluidas en el test final. El alumno se muestra satisfecho cuando sabe dar respuesta a una pregunta que no pudo contestar al inicio del tema. Igualmente, el test final sirve como sesión de repaso de los contenidos fijando los conceptos más importantes.

A través del Campus Virtual se informó, mediante recordatorios en el calendario, de los días previstos en que se iba a realizar la gamificación. Sorprendentemente la asistencia resultó del $100 \%$ de los matriculados a pesar de que la asistencia no era obligatoria y no había control por firmas. La justificación ante esta masiva asistencia no es otra que la recompensa, que puede suponer una mejora sustancial en la nota final de la evaluación.

Durante la actividad cada alumno contaba con su smartphone y competían de forma individual, dado que el número de alumnos matriculados no ha sido elevado en este curso académico. Para evitar que los alumnos con falta de conectividad o sin dispositivo estuvieran en desventaja, las sesiones se programaban en la sala de ordenadores, de forma que todos tenían acceso fijo o inalámbrico.

\subsection{Análisis de resultados y grado de satisfacción de los discentes}

A fin de valorar la experiencia educativa por parte de los alumnos se desarrolló una encuesta, voluntaria y anónima, cuyos ítems se evaluaban en una escala de 5 niveles de percepción (siendo 1-Muy malo y 5-Muy bueno). También se plantearon una serie de preguntas abiertas (ver Fig. 3). La encuesta fue completada por el 100\% de los alumnos, tras el último test de evaluación a través de KAHOOT!, dado que todos asistieron al aula.

Hemos constatado que la gamificación, que busca motivar convirtiendo el proceso de aprendizaje en algo lúdico, mejora los resultados de los alumnos. Las calificaciones en las pruebas de evaluación ordinaria mostraron una mejora significativa en los resultados respecto a convocatorias anteriores. Todos los alumnos superaron la evaluación. La continuación del uso de la metodología mostrará si en un futuro se sigue esta tendencia. 


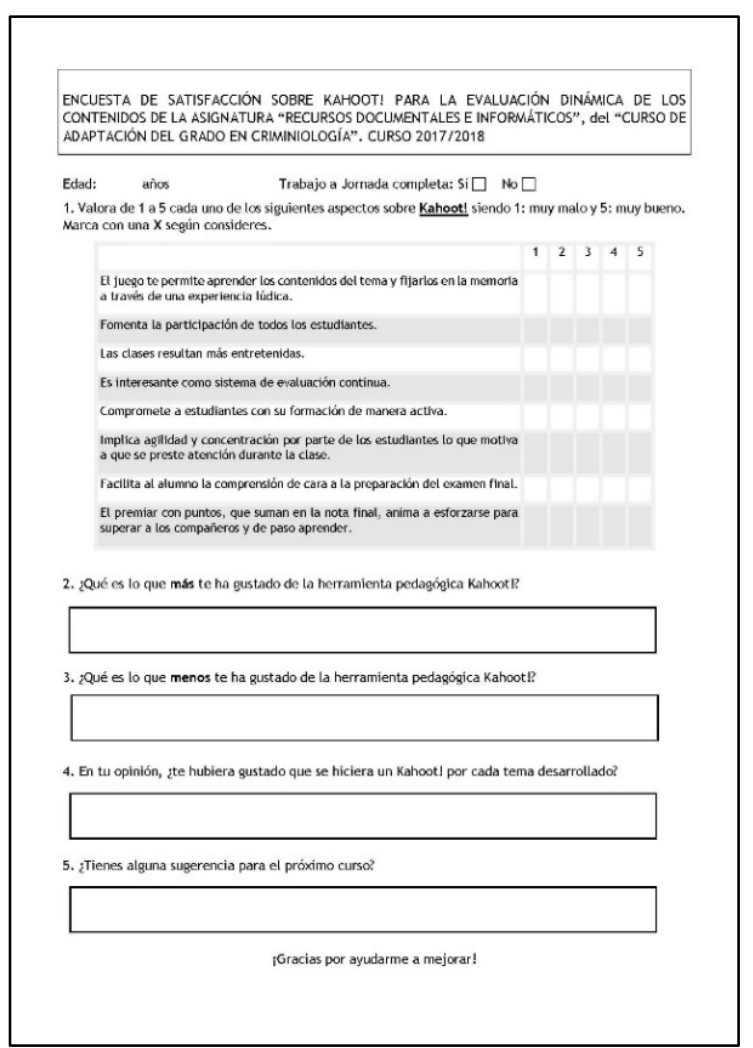

Fig. 3 Encuesta de satisfacción de la metodología de gamificación con KAHOOT!

\section{Resultados}

Los datos extraídos de las encuestas muestran la satisfacción por parte del alumno con esta actividad, que rompe con la linealidad de las clases magistrales.

Se les solicitó que valoraran las siguientes cuestiones:

1. El juego te permite aprender los contenidos del tema y fijarlos en la memoria a través de una experiencia lúdica.

2. Fomenta la participación de todos los estudiantes.

3. Las clases resultan más entretenidas.

4. Es interesante como sistema de evaluación continua.

5. Compromete a estudiantes con su formación de manera activa.

6. Implica agilidad y concentración por parte de los estudiantes lo que motiva a que se preste atención durante la clase.

7. Facilita al alumno la comprensión de cara a la preparación del examen final.

8. El premiar con puntos, que suman en la nota final, anima a esforzarse para superar a los compañeros y de paso aprender.

(c) EY-NC-ND 2018, Universitat Politècnica de València

Congreso IN-RED (2018) 
En la figura 4 se muestran los resultados. Los colores asignados a los niveles de percepción de la escala son: 1-Muy malo (azul claro), 2-Malo (morado), 3-Normal (verde), 4-Bueno (burdeos) y 5-Muy bueno (azul).

\begin{tabular}{|l|l|l|l|}
\hline CUESTIÓN 1 & CUESTIÓN 2 & CUESTIÓN 3 & CUESTIÓN 4 \\
\hline CUESTIÓN 5 & CUESTIÓN 6 & CUESTIÓN 7 & CUESTIÓN 8 \\
\hline
\end{tabular}

Fig. 4 Encuesta de satisfacción de la metodología de gamificación con KAHOOT!

La mayor parte de los alumnos puntuaron todas las preguntas con valores 4 y 5 . Solamente un alumno respondió con una valoración de 2 a la pregunta 7. Otro alumno respondió con 1 a la pregunta 8. Por tanto, ¡la metodología queda avalada!

La aplicación de esta metodología ha permitido:

- Valorar el nivel previo de conocimientos sobre la materia que tiene el alumno para ajustar el temario, la profundidad y rapidez de impartición de los contenidos, incidiendo en los puntos débiles de la materia.

- Evitar el absentismo gracias al hecho de otorgar reconocimiento, que se suma a la nota final de aquellos alumnos que han obtenido mejor calificación en los test. Los alumnos valoraron que era positivo poder sumar puntos de cara a la evaluación final.

- Aumentar la participación del alumno durante las clases, así como su atención, tan necesaria en la retención de los conceptos. La herramienta de evaluación muestra la respuesta correcta tras cada pregunta. Es eficaz que la transición entre preguntas las marque el docente, al poder plantear dudas en las pausas para clarificar conceptos y entender el porqué no se ha respondido correctamente a la cuestión (bien porque no entienden la pregunta o porque no saben la respuesta). Resulta interesante y didáctico exponer la pregunta de forma diferente para que entiendan conceptos que a priori pueden resultar abstractos. Es útil entablar un diálogo para aclarar los conceptos más importantes. Cuando los resultados sean negativos hay que explicar el motivo, de forma que resulte más sencillo asimilar conceptos de cara a la evaluación final. 
- Demostrar una mejora de los resultados académicos de los alumnos, tras el análisis de los datos obtenidos durante el curso y compararlos con los de años anteriores, cuando no se aplicaba la gamificación.

\section{Conclusiones}

Buscando la mejora de los resultados y la motivación de los alumnos, en este trabajo se plantea el uso de la ludificación, como didáctica encaminada a promover una enseñanza en la cual queda patente la comprensión y asimilación de los contenidos. La experiencia nos ha demostrado que la técnica de gamificación mejora los resultados de la evaluación final en comparación con los de cursos anteriores. Se trata de una dinámica integradora donde se compite de forma amable respondiendo a las preguntas que se plantean sobre la materia. Y es que en la gamificación las recompensas son elementos cruciales para el éxito, y más si suma en la nota final de la asignatura. También la sana competencia entre los estudiantes puede resultar un acicate para dedicar tiempo al estudio.

Gracias a los resultados de un sencillo test al inicio del tema, podemos conocer el nivel previo de conocimientos sobre la materia que tiene el alumno para ajustar el temario, la profundidad y rapidez de impartición de los contenidos e incidir en los puntos débiles.

El uso de incentivos, por obtener la mejor puntuación en relación al resto de compañeros en las pruebas de KAHOOT!, que se suman a la nota de la asignatura, claramente motiva y reduce el absentismo. La sola asistencia a la sesión es indicativa del interés de los alumnos.

La ingente cantidad de artículos y trabajos presentados en congresos demuestra el éxito de esta estrategia "amable", y puede animar a otros docentes con alumnos desmotivados. La realimentación proporcionada por alumnos permitirá los ajustes pertinentes, y el análisis de los datos extraídos, a través de las encuestas, mejorará la experiencia beneficiando al propio equipo implicado y, por supuesto, a los estudiantes.

Dada la eficacia de la metodología, en cursos posteriores se mantendrá en la asignatura de "Recursos Documentales e Informáticos" e implementará en otras asignaturas de grado, máster y doctorado.

\section{Agradecimientos}

Este trabajo no hubiera sido posible sin el apoyo proporcionado por el Vicerrectorado de Docencia de la Universidad de Valladolid (Proyecto de Innovación Docente No 134).

\section{Referencias}

BARNES, R. (2017). "Kahoot! in the Classroom: Student Engagement Technique” en Nurse Educator, vol. 42, issue 6, p. 280-280.

CHAIYO, Y.; NOKHAM, R. (2017). "The Effect of Kahoot, Quizizz and Google forms on the Student's Perception in the Classrooms Response System”. En: 2017 International Conference on

(cc) EY-NC-ND 2018, Universitat Politècnica de València

Congreso IN-RED (2018) 
Digital Arts, Media and Technology (ICDAMT) - Digital Economy for Sustainable Growth. pp. 178182. Chiang Mai.

FUERTES, A.; GARCÍA, M.; CASTAÑO, M. A.; LÓPEZ, E.; ZACARES, M.; COBOS, M.; FERRIS, R.; GRIMALDO, F. (2016). "Uso de herramientas de respuesta de audiencia en la docencia presencial universitaria. Un primer contacto”. En Actas de las XXII Jenui. pp. 262-268. Almería. Disponible en https://www.uv.es/grimo/publications/jenui2016.pdf [Consulta: 10 de marzo de 2018]

GARCIA-GARCIA, D.; CARBONELL-VERDU, A.; MONTAÑES, N.; QUILES, L.; FOMBUENA, V. (2017). "Incorporación de la aplicación Kahoot! para la evaluación de las prácticas de la asignatura de "Ciencia de Materiales”. Fernández Prada, M. Á. y Botti Navarro, V. J. (coord.) En: IN-RED 2017. Congreso nacional de innovación educativa y de docencia en red. pp. 1209-1217. Valencia: Editorial Universitat Politècnica de València. Disponible en http://hdl.handle.net/10251/86908 [Consulta: 15 de marzo de 2018]

GOOGLEFORMS <https://www.google.com/forms/about/> [Consulta: 3 de marzo de 2018]

IWAMOTO, D. H.; HARGIS, J.; TAITANO, E. J.; VUONG, K. (2017) “Analyzing the efficacy of the testing effect using Kahoot (TM) on student performance” en Turkish Online Journal of Distance Education, vol. 18, issue 2, p. 80-93.

KAHOOT!. <https://kahoot.com/> [Consulta: 26 de febrero de 2018]

LLERENA, E. G.; RODRIGUEZ, C. P. (2017) "Kahoot! A Digital Tool for Learning Vocabulary in a language classroom” en Revista Publicando, vol. 4, isuue 12, p. 441-449.

PEÑA, B.; ZABALZA, I.; USÓN, S.; LLERA, E. M.; MARTÍNEZ, A.; ROMEO L. M. (2017). "Experiencia piloto de aula invertida para mejorar el proceso de enseñanza-aprendizaje en la asignatura de TermodinámicaTécnica”. Fernández Prada, M. Á. y Botti Navarro, V. J. (coord.) En: IN-RED 2017. Congreso nacional de innovación educativa y de docencia en red. pp.583-596. Valencia: Editorial Universitat Politècnica de València. Disponible en http://hdl.handle.net/10251/86908 [Consulta: 14 de marzo de 2018]

PROYECTA (2018). “Gamificación: jugar para aprender”, http://www.plataformaproyecta.org/es/recursos-educativos/gamificacion-jugar-para-aprender [Consulta: 1 de marzo de 2018]

QUIZIZZ < https://quizizz.com/> [Consulta: 3 de marzo de 2018]

RODRIGUEZ-FERNANDEZ, L. (2017). "Smartphones and learning: use of Kahoot in the university classroom” en Revista Mediterranea Comunicacion-Journal of Communication, vol. 8, isuue 1, p. 181-189.

ROGER, S.; COBOS, M.; AREVALILLO-HERRÁEZ, M.; GARCÍA-PINEDA, M. (2017). "Combinación de cuestionarios simples y gamificados utilizando gestores de participación en el aula: experiencia y percepciónn del alumnado”. Fernández Prada, M. Á. y Botti Navarro, V. J. (coord.) En: IN-RED 2017. Congreso nacional de innovación educativa y de docencia en red. pp.1128-1139. Valencia: Editorial Universitat Politècnica de València. Disponible en http://hdl.handle.net/10251/86908 [Consulta: 16 de marzo de 2018]

SAN-MIGUEL, T.; MEGIAS, J.; SERNA, E. (2017). “Gamificación en la universidad II: aprendemos a divertirnos enseñando. Se divierten aprendiendo”. Fernández Prada, M. Á. y Botti Navarro, V. J. (coord.) En: IN-RED 2017. Congreso nacional de innovación educativa y de docencia en red. pp. 484-492. Valencia: Editorial Universitat Politècnica de València. Disponible en http://hdl.handle.net/10251/86908 [Consulta: 18 de marzo de 2018]

SOCRATIVE < https://www.socrative.com/> [Consulta: 3 de marzo de 2018]

(cc) EY-NC-ND 2018, Universitat Politècnica de València

Congreso In-Red (2018) 
SONG, Y. (2014). ““'Bring Your Own Device (BYOD)” for seamless science inquiry in a primary school” en Computers \& Education, vol. 74, p. 50-60.

TEJEDOR, F.J.; GARCÍA-VALCÁRCEL, A. (2007). "Causas del bajo rendimiento del estudiante universitario (en opinión de los profesores y alumnos). Propuestas de mejora en el marco del EEES” en Revista de Educación, vol. 342, p. 443-473.

WANG, A. I.; LIEBEROTH, A. (2016). "The Effect of Points and Audio on Concentration, Engagement, Enjoyment, Learning, Motivation, and Classroom Dynamics Using Kahoot!” En: Proceedings of the 10th European Conference on Games-Based Learning. pp. 738-746. Paisley.

(cc) EY-NC-ND 2018, Universitat Politècnica de València 